\title{
PROTEÇÃO DO ALUMÍNIO 2024 POR FILMES DE POLIPIRROL DEPOSITADOS EM ÁCIDO P-TOLUENO SULFÔNICO
}

\section{PROTECTION OF ALUMINUM 2024 BY POLYPYRROLE FILMS DEPOSITED IN P-TOLUENE SULFONIC ACID}

\author{
Karine Miranda Bezerra ${ }^{1}$ \\ Andréa Santos Liu $^{2}$ \\ Felipe Ezequiel Pereira dos Santos ${ }^{3}$ \\ Liu Yao $\mathrm{Cho}^{4}$
}

Resumo: Na presente pesquisa, investigou-se a eficiência dos filmes de Polipirrol (PPy), eletrodepositados potenciostaticamente em meio aquoso contendo Ácido p-Tolueno Sulfônico (pTS), para proteção da liga de alumínio 2024 contra a corrosão. Ensaios de Polarização Potenciodinâmica e de Voltametria Cíclica em meio corrosivo de cloreto mostraram que as superfícies recobertas com filmes de PPy/pTS, depositados a 1,2V, promoveram maior proteção da superfície metálica, quando comparado aos filmes de PPy/pTS, formados a 1,4V. A morfologia dos filmes foi investigada por Microscopia Eletrônica de Varredura (MEV), e as micrografias indicaram que os filmes de PPy eletrodepositados a 1,2V são mais homogêneos e uniformes, enquanto que os filmes formados a 1,4V apresentaram maior porosidade, o que favorece a adsorção de íons $\mathrm{Cl}$, que ocasionam a dissolução anódica das superfícies de alumínio.

Palavras-chave: corrosão; alumínio; polipirrol; ácido p-Toluenossulfônico.

Abstract: In this work, polypyrrole films were synthesized by electrochemical polymerization technique using aqueous medium containing pyrrole and p-toluenesulphonic acid. The efficiency of these polymeric films to protecting aluminum alloy against corrosion was investigated by potentiodynamic polarization curves. These results showed that the films deposited at $1.2 \mathrm{~V}$ perform better for protecting aluminum surfaces against corrosion. Furthermore, the influence of applied potential on the morphology of the films was studied by Scanning Electron Microscopy (SEM). The presence of pores in polymer deposited at $1.4 \mathrm{~V}$ alloy allowed the penetration of aggressive species that favors the corrosion process.

Keywords: corrosion; aluminum; polypyrrole; p-Toluenesulfonic acid.

\footnotetext{
${ }^{1}$ Graduanda em Química - Instituto Federal de São Paulo - IFSP, Brasil e Iniciação Científica - Universidade do Vale do Paraíba - Univap, Brasil. E-mail: karinemirb@gmail.com.

${ }^{2}$ Doutora em Engenharia Aeronáutica e Mecânica - Instituto Tecnológico de Aeronáutica - ITA, Brasil e Professora do IFSP, Brasil. E-mail: andrealiu1@gmail.com.

${ }^{3}$ Mestre em Processamento de Materiais e Catálise - Univap, Brasil. E-mail: felipeezequiel@ig.com.br.

${ }^{4}$ Doutor em Química - Universidade de São Paulo - USP, Brasil e Docente da Univap, Brasil. E-mail: liu@univap.br.
} 


\section{INTRODUÇÃO}

Desde 1930, as ligas de alumínio têm sido o material escolhido pelo setor aeronáutico, devido às suas excelentes propriedades, ressaltando-se a baixa massa específica (TAN: OGEL, 2007).

Compostos à base de cromo hexavalente atuam como excelente inibidor de corrosão de superfícies de alumínio, apresentando características atrativas, como elevada relação eficiência/custo, fácil aplicação, solubilidade em água, natureza passiva dos produtos formados na reação de oxirredução com o alumínio e alto poder oxidante (LIU, 2006). Entretanto, seu uso tem sido limitado, desde 1982, quando foram constatados seus efeitos carcinogênicos e mutagênicos (TWITE; BIERWAGEN, 1998). Dessa forma, novos sistemas têm sido estudados para proteção das superfícies de alumínio contra a corrosão.

A eficiência de polímeros condutores, como a polianilina (PAni) e o polipirrol (PPy), tem sido investigada na proteção de metais oxidáveis contra corrosão (TALLMAN et al., 2002; SPINKS et al., 2002). As propriedades físico-químicas do PPy, tais como estabilidade química/térmica, condutividade elétrica, baixa solubilidade do polímero em solventes orgânicos e em água, e a facilidade de síntese por métodos químicos e eletroquímicos o conferem destaque no campo da pesquisa (WANG; LI; YL, 2001; CASTAGNO; AZAMBUJA; DALMORO, 2009). A combinação dessas características com propriedades elétricas e ópticas levam o PPy à crescente aplicação tecnológica, destacando seu uso como material de eletrodo de supercapacitores (BAHLOUL et al., 2013; HASHMI; UPADHYAYA, 2002; TSAI; CHEN; DO, 2004), revestimento de compósito de nanotubos de carbono (HUGHES, et. al. 2002) e na proteção contra a corrosão (ZARRAS et al., 2003; TALLMAN et al., 2002; LIU et al., 2014). Na eletrodeposição de filmes de PPy, as propriedades elétricas, mecânicas e morfológicas do polímero são afetadas por inúmeros fatores experimentais, tais como $\mathrm{pH}$, eletrodo, temperatura, natureza e concentração do monômero/eletrólito, solvente e potencial de síntese (KAHALKHALI, 2005; SADKI et al., 2000).

Vale ressaltar que sulfonatos aromáticos podem atuar como dopantes para o polipirrol e influenciar nas propriedades eletroquimicas destes filmes, bem como na sua performance para proteger metais contra corrosão (WANG; DIARMID, 2001). Apesar de pesquisas retratarem a proteção de superficies de aço por filmes de PPy depositados em meio de sulfonato (GAUR; SRINIVASAN, 1999), poucos estudos abordam a proteção de ligas de alumínio por filmes de PPy depositados eletroquimicamente em meio de ácido sulfônico. Estudos prévios realizados em nosso laboratorio mostraram que filmes de PPy depositados galvanostaticamente, foram capazes de proteger o aluminio 99,9\% contra corrosão.

Neste trabalho, é retratada a proteção da liga de alumínio 2024, empregada no setor aeronáutico, por filmes de PPy eletrodepositados potenciostaticamente em meio aquoso de ácido p-toluenossulfônico (pTS). 


\section{EXPERIMENTAL}

A Figura 1 apresenta um fluxograma das etapas envolvidas no desenvolvimento deste trabalho.

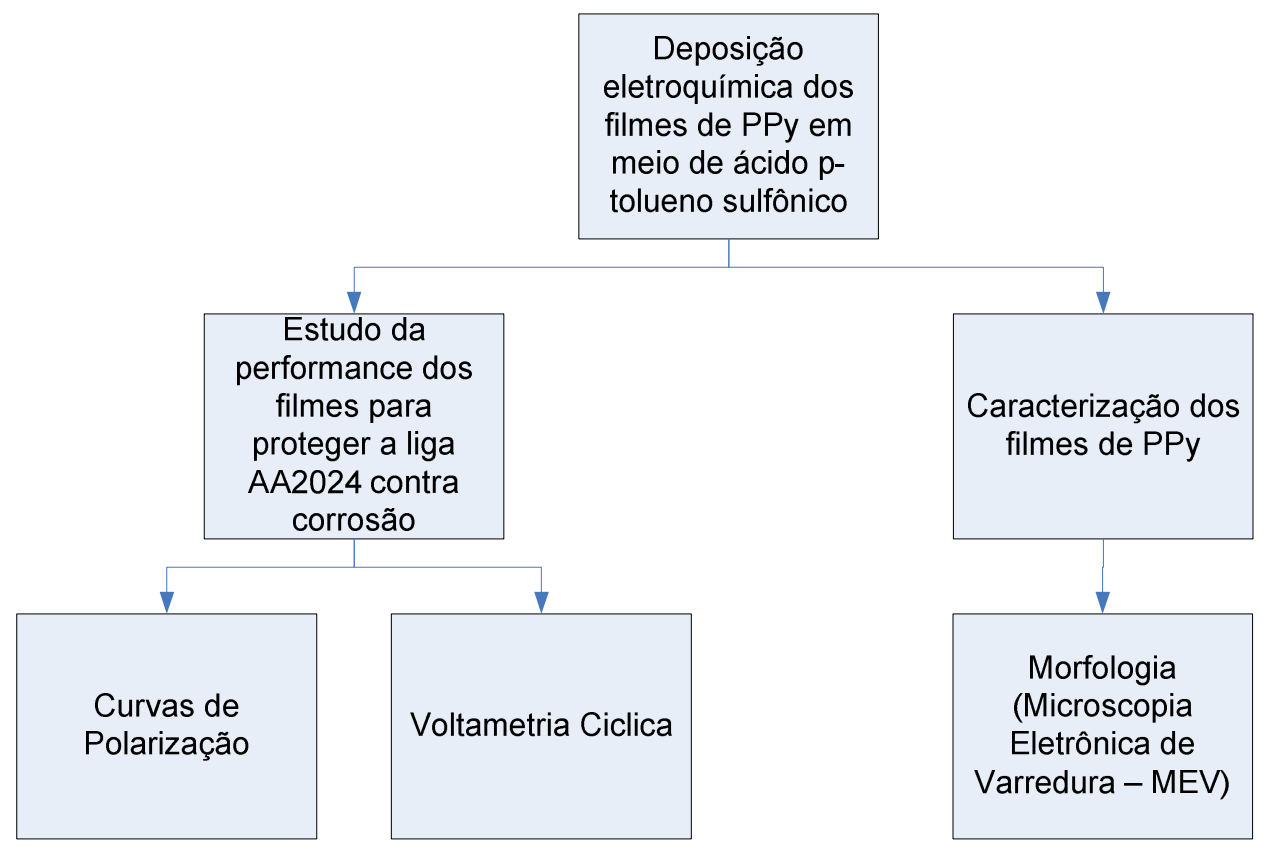

Figura 1 - Fluxograma das etapas desenvolvidas no trabalho.

\subsection{Reagentes, Soluções e Materiais}

A liga de alumínio 2024 é constituída por 4,4\% de cobre, 1,5\% de magnésio e 0,6\% de manganês. A eletrodeposição dos filmes de PPy sobre a liga metálica foi realizada em meio aquoso contendo Pirrol (Py, 0,2 mol. $\mathrm{L}^{-1}$ ) e Ácido p-Toluenossulfônico (pTS, 0,1 mol.L-1). Em cada experimento eletroquímico, a superfície metálica foi polida com lixas com granulometria de 360, 600 e 1200, respectivamente. Realizou-se, na sequência, um polimento com suspensão aquosa de alumina e enxágue com água deionizada.

Os ensaios eletroquímicos foram realizados em uma célula contendo três eletrodos: $(I)$

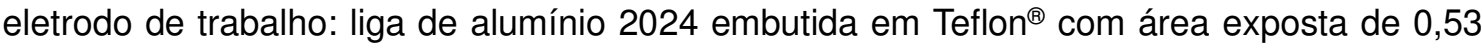
$\mathrm{cm}^{2}$; (ii) eletrodo auxiliar: fio de platina e (iii) eletrodo de referência: eletrodo de $\mathrm{Ag} / \mathrm{AgCl}, \mathrm{Cl}^{-}$ saturado.

\subsection{Eletrodeposição de Filmes de PPy e Ensaios de Polarização Potenciodinâmica}

Os filmes de PPy foram depositados a potencial controlado e à temperatura ambiente, utilizando-se um Potenciostato/Galvanostato, modelo MQPG-01 Microquímica, conectado e controlado por um microcomputador. A influência do eletrólito foi investigada 
potenciostaticamente, utilizando-se solução aquosa 0,2 mol.L-1 de pTS. A eletrodeposição dos filmes foi averiguada aplicando-se potencial em $+1,2 \mathrm{e}+1,4 \mathrm{~V} v s$. $\mathrm{Ag} / \mathrm{AgCl}$, durante $900 \mathrm{~s}$.

Os ensaios de polarização potenciodinâmica foram realizados em solução aquosa de $\mathrm{NaCl}$ 0,1 mol.L-1 (pH 5,9), variando-se o potencial de $-1,5 \mathrm{a}+1,5 \mathrm{~V}$ a $5 \mathrm{mV} . \mathrm{s}^{-1}$. Os valores de potencial de corrosão $\left(E_{\text {corr }}\right)$ e de densidade de corrente de corrosão ( $\left.j_{\text {corr }}\right)$ foram obtidos a partir das curvas de Tafel; sendo o $\mathrm{E}_{\text {corr }}$ determinado pela intersecção dos ramos anódicos e catódicos quando $\mathrm{j}=0$, e os valores de jorr extrapolados no potencial de corrosão por intermédio da intersecção dos trechos lineares das curvas.

\subsection{Caracterização dos Filmes de PPy}

A morfologia das superfícies de alumínio recobertas com filmes de PPy/pTS e os espectros de EDS foram analisados utilizando-se um Microscópio Eletrônico de Varredura (MEV), modelo Jeol JXA 840A. As micrografias foram obtidas utilizando-se um feixe de elétrons de $15 \mathrm{keV}$.

\section{RESULTADOS E DISCUSSÃO}

\subsection{Eletrodeposição dos Filmes de PPy/pTS}

Inicialmente, os filmes de PPy foram depositados por voltametria cíclica, variando-se o potencial entre $-1,5 \mathrm{~V}$ a $+1,5 \mathrm{~V}$, a $5 \mathrm{mV}^{-1}$. A Figura 2 apresenta os voltamogramas para as superfícies de alumínio expostas a soluções eletrolíticas contendo: (1) 0,2 mol.L-1 de Py e 0,1 mol.L-1 de pTS e (2) 0,1 mol.L-1 de pTS.

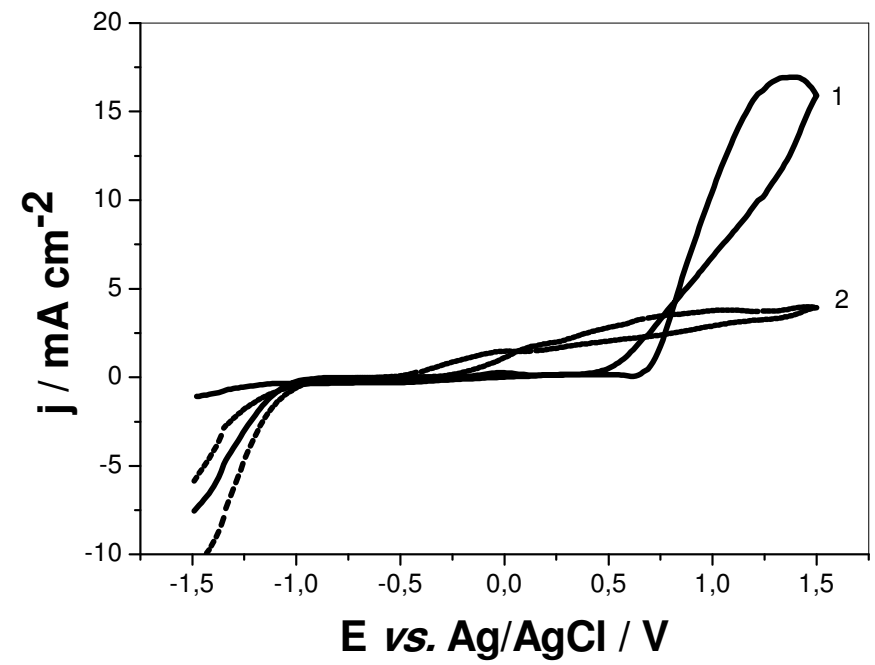

Figura 2 - Voltamograma cíclico para: (1) Eletrodeposição de PPy sobre a superfície de alumínio utilizando-se solução aquosa contendo 0,2 mol.L-1 de Py e 0,1 mol.L-1 de pTS e (2) Influência do ácido pTS sobre o substrato metálico. 
A Curva 1 (Figura 2) apresenta um pico anódico à, aproximadamente, 1,4V, que deve estar associado à oxidação do pirrol (Py). Vale ressaltar que a ausência de um pico catódico neste voltamograma indica que o processo de eletropolimerização do Py é irreversível. A irreversibilidade na eletrodeposição dos filmes de PPy é similar à obtida na deposição de filmes de PPy/pTS sobre superfícies de alumínio 99,9\% (LIU; BEZERRA; CHO, 2009). Já, as correntes anódicas, observadas no voltamograma da Curva 2, estão associadas à oxidação do metal em meio de ácido pTS. Esse resultado é corroborado pelo ensaio de Transiente de I $x$ t, apresentado na Figura 3, para as superfícies de alumínio na ausência de Py.

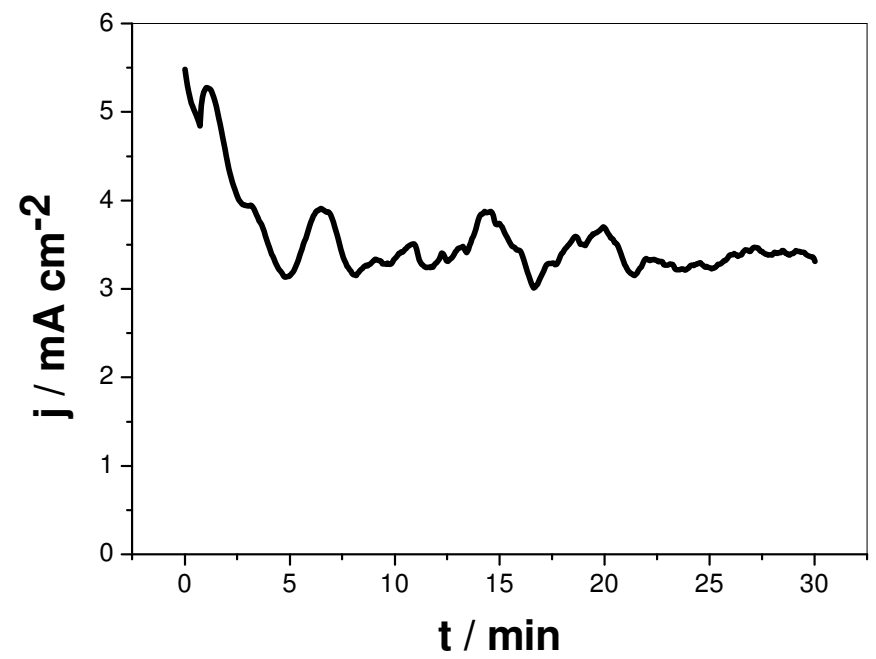

Figura 3 - Transiente de corrente versus tempo para a exposição das superfícies de alumínio ao ácido pTS.

As oscilações de correntes, nas curvas de potencial versus tempo, podem estar associadas à formação de pitting metaestáveis na superfície metálica (LIU; OLIVEIRA, 2007).

A formação de filmes de PPy sobre substratos de alumínio considera a constituição de uma estrutura sanduíche do tipo $\mathrm{Al} / \mathrm{Al}_{2} \mathrm{O}_{3} / \mathrm{PPy}$ (NAOI, et. al. 2000). A natureza do eletrólito influencia na deposição dos filmes poliméricos. Estudos indicam que a porosidade na camada de óxido de alumínio serve como sítios ativos para o crescimento do filme polimérico (VILCA; MORAES; MOTHEO, 2004).

As curvas de corrente versus tempo para a deposição de PPy em meio aquoso constituído de 0,2 mol.L-1 de Py e 0,1 mol.L-1 do ácido pTS, aplicando-se potencial de 1,2 e $1,4 \mathrm{~V}$, são apresentadas na Figura 4. 


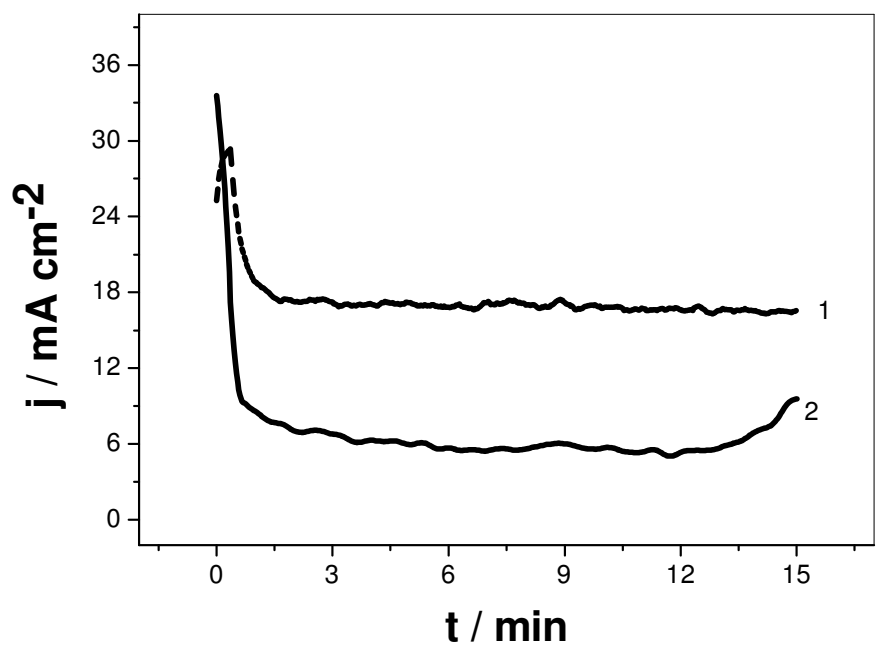

Figura 4 - Curvas de corrente versus tempo para a eletrodeposição de filmes de PPy em solução aquosa contendo $0,2 \mathrm{~mol}^{-\mathrm{L}^{-1}}$ de Py e $0,1 \mathrm{~mol} . \mathrm{L}^{-1}$ de $\mathrm{pTS}$, aplicando-se potencial de: (1) $1,2 \mathrm{~V}$ e (2) $1,4 \mathrm{~V}$ durante $900 \mathrm{~s}$.

O comportamento das curvas 1 e 2 da Figura 4 mostraram inicialmente um brusco crescimento de corrente, seguido pela queda exponencial da corrente, associada à nucleação e deposição dos filmes de PPy/pTS sobre o eletrodo de alumínio. As curvas de $J x t$ mostraram que as densidades de corrente associadas ao crescimento dos filmes de PPy são maiores quando foi aplicado 1,2V em relação a 1,4V. Este resultado foi atribuído a maior condutividade do filme PPy depositado nesta condição.

\subsection{Ensaios de Corrosão: Curvas de Polarização Potenciodinâmica}

As curvas de polarização para os filmes de PPy depositados em meio aquoso de ácido pTS no potencial de 1,2V e 1,4V são apresentadas na Figura 5.

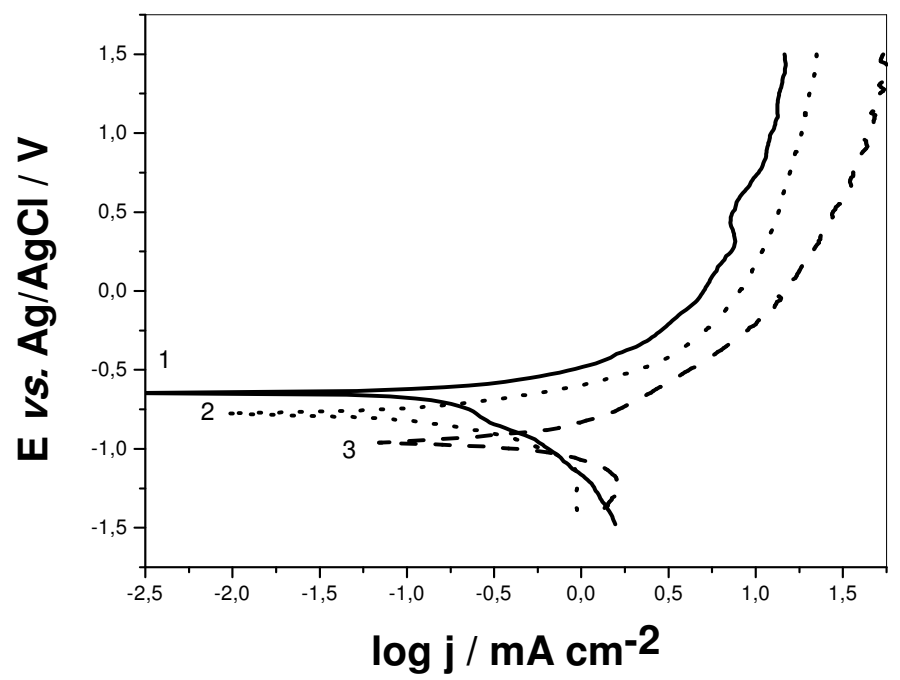

Figura 5 - Curvas de Tafel obtidas em meio de $\mathrm{NaCl}\left(0,1 \mathrm{~mol} . \mathrm{L}^{-1}\right)$ para as superfícies de alumínio recobertas com filmes de PPy depositados durante 900 s com aplicação de potencial de: (1) $1,2 \mathrm{~V}$; (2) 1,4V e (3) Apenas polida. A velocidade de varredura de potencial foi de $5 \mathrm{mV} . \mathrm{s}^{-1}$. 
Os parâmetros de corrosão obtidos a partir das curvas de Tafel são expostos na Tabela 1.

Tabela 1 - Parâmetros de corrosão obtidos para os substratos de alumínio polarizados em $\mathrm{NaCl}$

\begin{tabular}{lcc}
\hline \multicolumn{1}{c}{ Superfícies de Alumínio } & $\mathbf{E}_{\text {corr }} \mathbf{V s} . \mathbf{A g} / \mathbf{A g C l} / \mathbf{V}$ & $\mathbf{j}_{\text {corr }} / \mathbf{~ m A ~ c m}^{-2}$ \\
\hline Apenas polida & $-0,96$ & 0,65 \\
Recoberta com PPy/pTS a 1,2V & $-0,65$ & 0,25 \\
Recoberta com PPy/pTS a 1,4V & $-0,77$ & 0,30 \\
\hline
\end{tabular}

Mediante análise das curvas de Tafel e dos parâmetros de corrosão, pode ser observado um deslocamento do potencial de corrosão, $E_{\text {corr, }}$ para a direção positiva, quando as superfícies de alumínio estão recobertas com filmes de PPy. Considerando-se que a densidade de corrente de corrosão, jcorr, está diretamente associada à velocidade de corrosão do metal, pode-se inferir que as superfícies de alumínio recobertas com filmes de PPy eletrodepositados a 1,2V apresentam melhor desempenho na proteção contra a corrosão. As possíveis reações envolvidas durante os ensaios de polarização das superfícies de alumínio são expressas pelas Equações (LIU, 2006):

Reações Anódicas:

$$
\begin{aligned}
\mathrm{Al}_{(\mathrm{s})} & \rightarrow \mathrm{Al}^{3+}{ }_{(\mathrm{aq})}+3 \mathrm{e}^{-} \\
\mathrm{PPy}_{\text {desdopado }} & \rightarrow \mathrm{PPy}_{\text {dopado com } \mathrm{Cl}^{-}+\mathrm{n} \mathrm{e}^{-}}
\end{aligned}
$$

Reações Catódicas:

$$
\begin{gathered}
2 \mathrm{H}_{2} \mathrm{O}_{(\mathrm{l})}+\mathrm{O}_{2}(\mathrm{~g})+4 \mathrm{e}^{-} \rightarrow 4 \mathrm{OH}_{(\mathrm{aq})}^{-} \\
\mathrm{PPy} \text { dopado }+\mathrm{n} \mathrm{e}^{-} \rightarrow \mathrm{PPy}_{\text {desdopado }}
\end{gathered}
$$

Os resultados de voltametria cíclica em meio de $\mathrm{NaCl}$ para as superfícies de alumínio recobertas com filmes de PPy/pTS depositados em 1,2 e 1,4V são apresentados na Figura 6.

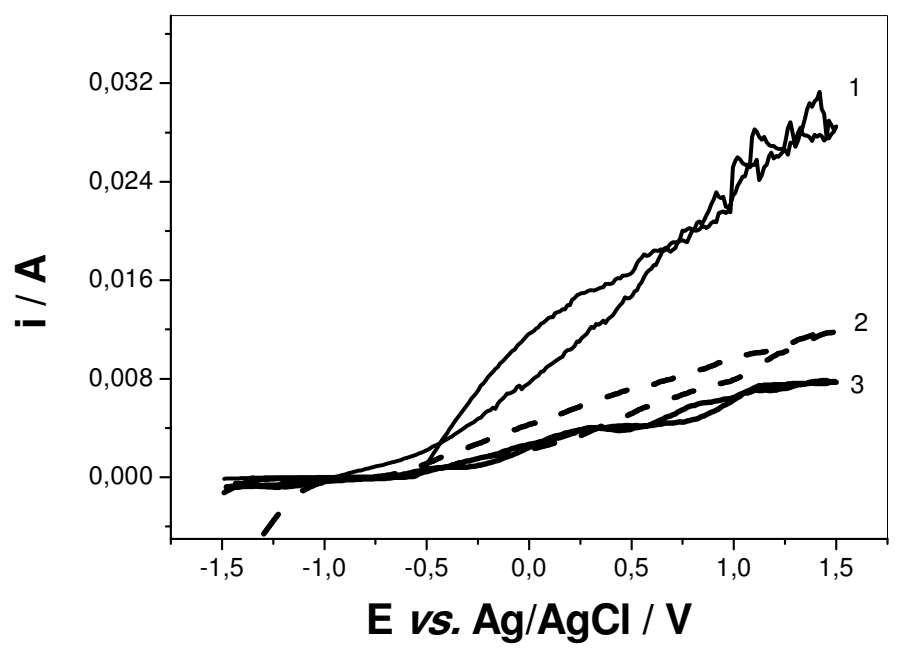

Figura 6 - Voltametria cíclica obtida em meio de $\mathrm{NaCl}\left(0,1 \mathrm{~mol}^{-\mathrm{L}^{-1}}\right)$ para as superfícies de alumínio: (1) Apenas polida; Recobertas com filmes de PPy/pTS depositados durante $900 \mathrm{~s}$ aplicando-se potencial de: (2) $1,4 \mathrm{~V}$ e (3) $1,2 \mathrm{~V} . \mathrm{v}=5 \mathrm{mV} . \mathrm{s}^{-1}$. 
Analisando-se os voltamogramas (Figura 6), podem ser observados menores valores de corrente para as superfícies de alumínio recobertas com filmes de PPy/pTS depositados a $1,2 \mathrm{~V}$, indicando a melhor condição de proteção do alumínio 2024 contra a corrosão. Considerando-se que a adsorção de íons $\mathrm{Cl}^{-}$cresce linearmente com o aumento do potencial (SMIALOWSHA-SZKLARSKA, 1999), pode-se inferir que os filmes de PPy depositados em $1,2 \mathrm{~V}$ demonstram comportamento protetor mesmo em elevados potenciais.

\subsection{Caracterização dos Filmes de PPy}

A Morfologia dos filmes de PPy eletrodepositados potenciostaticamente em meio contendo o ácido pTS foi investigada por Microscopia Eletrônica de Varredura (MEV). A Micrografia das superfícies de alumínio revestidas com filmes de PPy/pTS eletrodepositados em 1,2 e 1,4V é apresentada na Figura 7.

(a)

(b)

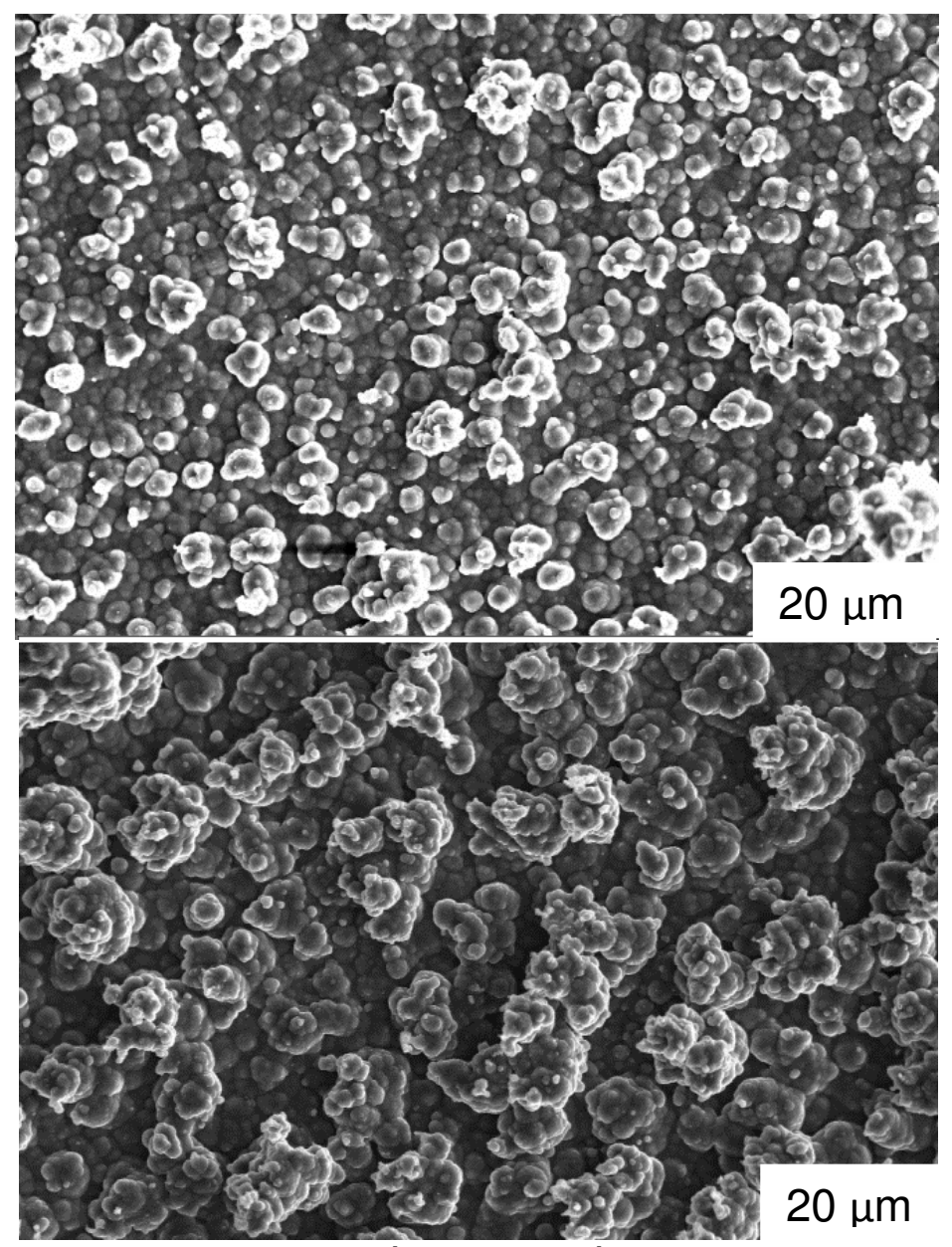

Figura 7 - Micrografia (MEV) das superfícies de alumínio recoberta com filmes de PPy/pTS depositado durante 900 s aplicando-se potencial de: (a) 1,2V e (b) 1,4V. 
A Morfologia dos filmes de PPy/pTS apresentou uma estrutura do tipo cauliflower, constituída por grãos micro-esféricos, que tem sido atribuída à dificuldade de intercalação do dopante na cadeia polimérica desordenada (BAZZAOUI, et al., 2002); os filmes de PPy/pTS depositados a 1,2V são mais homogêneos e compactos, o que pode estar relacionado com a melhor performance desses filmes na proteção do alumínio 2024 contra a corrosão.

Os espectros de Energia Dispersiva de Raio-X (EDS) (Figura 8) indicam a presença de átomos de enxofre na cadeia polimérica, resultante da incorporação dos ânions do pTS durante a deposição eletroquímica dos filmes de PPy.

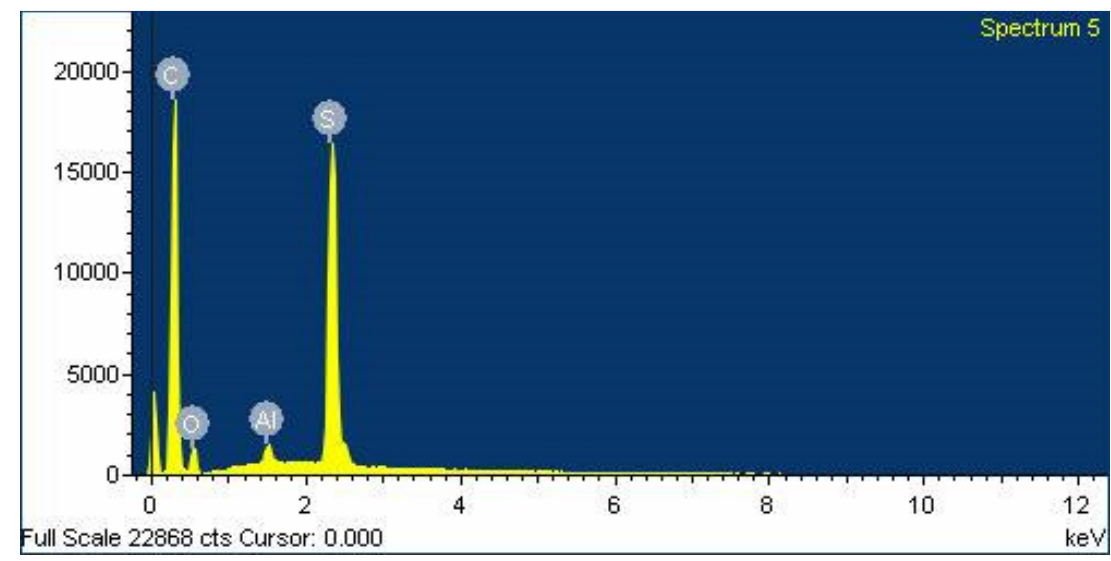

Figura 8 - Espectro EDS dos filmes de PPy eletrodepositados em 1,2V.

Além disso, vale ressaltar que estudos prévios desenvolvidos em nosso laboratório mostram que os espectros de Infravermelho (FTIR), para o filme de PPy depositado em superfícies de alumínio 99,9\%, em meio de pTS, apresentaram bandas de absorção a: 1635 $\mathrm{cm}^{-1}$ (deformação axial $\mathrm{C}=\mathrm{C}$ ), $1302 \mathrm{~cm}^{-1}$ (deformação axial assimétrica $\mathrm{O}=\mathrm{S}=\mathrm{O}$ ) e $1132 \mathrm{~cm}^{-1}$ (deformação axial simétrica $\mathrm{O}=\mathrm{S}=\mathrm{O}$ ), sugerindo que o ácido $\mathrm{p}$-tolueno sulfônico foi incorporado no polímero como dopante (LIU; BEZERRA; CHO, 2009).

\section{CONCLUSÃO}

Os filmes de PPy foram depositados sobre a liga de alumínio 2024 por meio da voltametria cíclica e por cronoamperometria. Foi observado que o potencial aplicado para eletropolimerizar o pirrol influencia na homogeneidade do filme polimérico e na sua performance para proteger a liga metálica contra corrosão. Os ensaios de Polarização Potenciodinâmica, em meio corrosivo de cloreto, mostraram um deslocamento do potencial de corrosão para a direção positiva e menores densidades de corrente de corrosão para as superfícies de alumínio recobertas com PPy em relação ao metal apenas polido. Dessa forma, pode ser inferido que os filmes poliméricos foram capazes de proteger a liga de alumínio 2024 contra corrosão. Os ensaios de Voltametria Cíclica confirmaram esse resultado, apresentando 
menores densidades anodicas associadas com a oxidação do metal, quando as superfícies foram revestidas com PPy. Além disso, ressalta-se que os parâmetros eletroquímicos indicaram maior proteção do alumínio 2024 quando revestido pelos filmes de PPy/pTS formados a $1,2 \mathrm{~V}$.

A Morfologia mostrou que os filmes de PPy depositados a 1,2V são mais compactos com maior homogeneidade e uniformidade do que a $1,4 \mathrm{~V}$, o que foi relacionado com o melhor desempenho desses filmes na proteção do metal contra corrosão. Esses filmes poliméricos também apresentaram a incorporação de anion do ácido p-TS na cadeia.

\section{REFERÊNCIAS}

BAHLOUL, A. et al. Polypyrrole-covered $\mathrm{MnO}_{2}$ as electrode material for supercapacitor. Journal of Power Sources, v. 240, pp. 267-272, 2013.

BAZZAOUI, M. et al. New single-step electrosynthesis process of homogeneous and strongly adherent polypyrrole films on iron electrodes in aqueous medium. Electrochimica Acta, v. 47, pp. 2953-2962, 2002.

CASTAGNO, K.; AZAMBUJA, D.; DALMORO, V. Polypyrrole electropolymerized on aluminum alloy 1100 doped with oxalate and tungstate anions. Journal of Applied Electrochemistry, v. 39, pp. 93-100, 2009.

GAUR, B.; SRINIVASAN, H. S. Corrosion of metals and alloys in methane sulphonic acid. British Corrosion Journal, v. 34, n. 1, pp. 63-66, 1999.

HASHMI, S. A.; UPADHYAYA, H. M. Polypyrrole and poly(3-methyl thiophene)-based solid state redox supercapacitors using ion conducting polymer electrolyte. Solid State Ionics, v. 152-153, pp. 883-889, 2002.

HUGHES, M. et al. Electrochemical capacitance of a nanoporous composite of carbon nanotubes and polypyrrole. Chemistry of Materials, v. 14, pp. 1610-1613, 2002.

KAHALKHALI, R. A. Electrochemical synthesis and characterization of electroactive conducting polypyrrole polymers. Russian Journal of Electrochemistry, v. 41, pp. 10711078, 2005.

LIU, A. S. Comportamento de superfícies de alumínio recobertas com filmes de polipirrol. 2006. Tese (Doutorado em Ciências - Física e Química dos Materiais Aeroespaciais) - Instituto Tecnológico de Aeronáutica, São José dos Campos, São Paulo, 2006.

LIU, A. S.; OLIVEIRA, M. A. S. Electrodeposition os polypyrrole films on aluminum from tartrate aqueous solution. Journal of the Brazilian Chemical Society, v. 18, pp. 143-152, 2007.

LIU, A. S.; BEZERRA, M. C.; CHO, L. Y. Electrodeposition of polypyrrole films on aluminum surface from a p-toluene sulfonic acid medium. Materials Research, v. 12, pp. 503-507, 2009.

LIU, A. S. et al. Electrodeposition of polypyrrole films on 2024 aluminum alloy in phosphoric 
acid solution. Materials Science Forum, v. 775-776, pp. 225-229, 2014.

$\mathrm{NAOI}, \mathrm{K}$. et al. Simultaneous electrochemical formation of $\mathrm{Al}_{2} \mathrm{O}_{3} /$ polypyrrole layers (I): effect of electrolyte anion in formation process. Electrochimica Acta, v. 45, pp. 3413-3421, 2000.

SADKI, S. et al. The mechanisms of pyrrole electropolymerization. Chemistry Society Reviews, v. 29, pp. 283-293, 2000.

SMIALOWSHA-SZKLARSKA, Z. Pitting corrosion of aluminum. Corrosion Science, v. 41, pp. 1743-1767, 1999.

SPINKS, G. M. et al. Electroactive conducting polymers for corrosion control - part 2: ferrous metals. Journal Solid State Electrochemistry, v. 6, pp. 85-100, 2002.

TALLMAN, D. E. et al. Electroactive conducting polymers for corrosion control - part 1: general introduction and a review of non-ferrous metals. Journal Solid State Electrochemistry, v. 6, pp. 73-84, 2002.

TAN, E.; OGEL, B. Influence of heat treatment on the mechanical properties of AA6066 alloy. Turkish Journal of Engineering e Environmental Sciences, v. 31, pp. 53-60, 2007.

TSAI, M. L.; CHEN, P. J.; DO, J. S. Preparation and characterization of $\mathrm{PPy} / \mathrm{Al}_{2} \mathrm{O}_{3} / \mathrm{Al}$ used as a solid-state capacitor. Journal of Power Sources, v. 133, pp. 302-311, 2004.

TWITE, R.; BIERWAGEN, G. Review of alternatives to chromate for corrosion protection of aluminum aerospace alloys. Progress in Organic Coatings, v. 33, pp. 91-100, 1998.

VILCA, D. H.; MORAES, S. R.; MOTHEO, A. J. Anodic treatment of aluminum in nitric acid containing aniline, previous to deposition of polyaniline and its role on corrosion. Synthetic Metals, v. 140, pp. 23-27, 2004.

WANG, L.; LI, X.; YL, Y. Preparation, properties and applications of polypyrroles. Reactive e Functional Polymers, v. 47, pp. 125-139, 2001.

WANG, P. C.; DIARMID, A. G. Dependency of properties of in situ deposited polypyrrole films on dopant anion and substrate surface. Synthetic Metals, v. 119, pp. 367-368, 2001.

ZARRAS, P. et al. Progress in using conductive polymers as corrosion-inhibiting coatings. Radiation Physics and Chemistry, v. 68, pp. 387-394, 2003. 